\title{
Clinical trials in relapsing-remitting multiple sclerosis (a new proposal for dealing with basic problems and restrictions)
}

\author{
Mieczyslaw Wender', Grazyna Michalowska-Wender ${ }^{1,2}$ \\ ${ }^{1}$ Neuroimmunological Unit, Mossakowski Medical Research Centre, Polish Academy of Sciences \\ ${ }^{2}$ Laboratory of Neurogenetics, Department of Neurology, Poznan University of Medical Sciences, Poland
}

\begin{abstract}
The natural course of multiple sclerosis is characterized by a high variability of pattern, relapse rate and different progression indices. They also present a dramatic impact on the interpretation of treatment trials. Older reports, based on uncontrolled observations are therefore of little value. Currently it is generally accepted that a proper treatment trial should be double blinded and, although probably controversial, that it should be compared with a group of MS patients treated with placebo. Such an approach would be easily acceptable to prove the effectiveness of recently discovered disease modifying drugs. We know that current standard methods of therapy with interferon beta or glatiramer acetate are able to decrease the relapse rate at least by one third, to elongate the intervals between the relapses and to decrease the progression indices. Currently MS is considered a generalized degenerative disease. The lesions are persistent, therefore immunomodulatory treatment has to be started as early as possible. An alternative approach, somewhat suggestive for the use of placebo trials, seems to be a comparison of proposed new drug therapy group with a group of patients treated with a generally accepted reference drug, such as interferon beta or glatiramer acetate, including all clinical and MRI measures.
\end{abstract}

Keywords: multiple sclerosis, clinical trial, placebo, pathophysiology, reference drug.

\section{Introduction}

Multiple sclerosis (MS) is a subacute inflammatory disease invading the central nervous system (CNS) of young adults, with presumably $T$ cell-mediated autoimmune pathogenesis. The incidence and prevalence of MS is significantly higher in females than in males. The reason for such a gender difference remains still unknown.

The natural cause of MS is characterized by variability of pattern, relapse rate and progression indices. In many patients there is a tendency to improve even without treatment. These facts create a dramatic impact on the interpretation of treatment trials. Older reports, based on uncontrolled observations were critically presented in a monography on therapeutic claims in MS [1] and are of less current value. The spontaneous remissions were often presented with great enthusiasm in older reports. Nevertheless, when the same method was replicated in other locations, results seemed not better than no therapy. This possibly means that results of the proposed new therapy were only describing the natural course of MS.

It would not be difficult to judge the efficiency of a new treatment when the marked improvement would occur in all cases or more importantly, when completely stopping the progress of the disease. So far, newly proposed methods were partially effective and therefore may only be evaluated by proper methods of clinical trials.

The first currently accepted rule is that the trial should be double blinded, from both patients and phy- 
sicians in order to avoid over enthusiasm with regard to improvement. In a good clinical trial, the team has to be strictly divided into the evaluating and treating physicians who are to guard the safety of the patients during the trial.

Multicentre trials usually provide a larger number of subjects and avoid bias related to ethnic or geographical differences. MS is most common in Caucasians living far away from the equator in the northern and southern hemispheres.

The results of treatment are evaluated by clinical and imaging techniques, such as structural MRI (magnetic resonance imaging), with or without gadolinium enhancement. In multicentre trials standardization based on exact definitions is required. The clinical status of the patient is assessed using a scoring system, e.g. the functional systems score (FS) included in the expanded disability status scale (EDSS) Kurtzke [2]. Intrarater and interrater reliability of the EDSS scale is sufficient. Aditionally, the multiple sclerosis functional composite (MSFC) is used [3]. MSFC test, the ambulation (timed 25-foot walk), arm function (NineHole Peg Test) and cognitive function (Paced Auditory Serial Addition Test (PASAT)). EDSS and MSFC scoring, when repeated in the course of trial, make it possible to establish whether or not the disease is progressing, has stopped or ameliorates. The use of scales yields also a possibility to establish objectively the occurrence of relapses or clinical deterioration. It is, actually, more complicated than a mere scoring matter, i.e. McDonald's diagnosis criteria are used.

A strict definition of relapse is used, i.e. the appearance of a new neurological abnormality or worsening of an existing one, separated by at least 30 days from a the previous attack A relapse should be confirmed by an evaluating physician. Confirmation is based on increase by 1 point in each of two functional systems (FS) or by 2 points in one.

Based on the agreed definitions and scoring systems, the number of relapse-free patients, the number of patients with treated relapses, the annual relapse rate and the number of patients with sustained clinical worsening is calculated within each trial.

Clinical results are compared with MRI lesions seen on repeated scans. The main MRI parameters include the number and volume of new and newly enlarging $\mathrm{T} 2$ hyperintensive and $\mathrm{T} 1$ hypointensive lesion [4, 5]. A measure of the volume of brain parenchymal fraction as an indication of brain atrophy is also performed [6].

One point in the planning of clinical trials in MS is both exciting and controversial at the same time.
Should the group of patients randomized to the effective studied drug be compared with another one, receiving placebo. Such an approach was easily acceptable to prove the effectiveness of disease modifying drugs. We know that current standard methods of therapy with interferon beta or glatiramer acetate are capable to decrease at least by one third the relapse rate, to elongate the intervals between the relapses as well as to decrease the progression indices based on the EDSS scale [7]. The impact of drug therapy on MRI lesions in the course of the process is even greater [4]. The idea of placebo-controlled clinical trials in MS was thoroughly discussed by international task force groups of clinicians, statisticians and ethicist and conclusions were published in 2001 and 2009 as a commonly reached consensus [8]. The conclusion was, that the placebo controlled clinical trials are acceptable from an ethical point of view, but only when the respective patients are fully aware of the overall available treatment procedures, or when they have already failed on previous therapy, or else, when for various reasons, they have no access to other treatment methods $[9,10]$.

However, quite recently, some new facts have been revealed in the field of pathophysiology of MS, which shadowed with dark clouds the placebo-controlled clinical trials in MS.

Currently, MS is considered as a degenerative disease of the brain and spinal cord in which an inflammatory process leads early not only to demyelinating lesions but also to an injury of axons, to neuronal loss and cerebral atrophy $[6,11]$. The lesions are persistent, which is the reason why immunomodulatory treatment has to be started as soon as possible [12].

Early axonal lesions were seen using immunohistochemical methods, such as the SMI-22 antibody detection method [13]. Normal, myelinated axons contain phosphorylated neurofilaments which are not stained bySMI-22. In acute MS lesions, in normal appearing white matter and even in the grey matter of post-mortem MS specimens, numerous dendrites with non-phosphorylated neurofilaments are found, thus showing axonal dysfunction. The presence of terminal axonal ovoids is proof of tissue transsection. Similar observations were made using other axonal protein marker of amyloid precursor.

Magnetic resonance tomography using T1-weighted (T1W) and T2-weighted (T2W) protocols shows atrophy of the grey matter, particularly within the cerebral cortex. It occurs early in the evolution of MS, and is of high clinical significance, triggering several symptoms and signs of the disease [14-17]. Recently evi- 
dent severe lesions were also found within the lower motor neurons of the spinal cord. Early immunomodulatory treatment can stop the progress of degenerative changes [12]. This does not happen in patients receiving placebo, even when an active treatment would later follow. To avoid ethical criticism related to the delay of active treatment, some protocols have limited the duration of the study to a 6 -months period, followed by open label treatment with the active drug only for the patients group.

An alternative approach, indicative of the rational value of placebo trials seems to be a comparison of the patients group receiving the new drug with a matched group treated with the reference drug of general acceptance e.g. interferon beta or glatiramer acetate, and assessment of subsequent clinical and MRI changes.

I understand that the presented point of view stands against the accepted dogma about the value of placebo control trials in MS. However, the numerous limitations raised by ethical principles against the use of placebo in MS trials, raised by Ethics Committees protecting those principles may lead to a creation of special groups of treated patients, that are not identical with the total cohort of MS patients. In this sense, the use of reference treated groups may even improve the validity of results.

\section{Acknowledgements}

\section{Conflict of interest statement}

The authors declare that there is no conflict of interest in the authorship or publication of contribution.

\section{Funding sources}

There are no sources of funding to declare.

\section{References}

1. Sibley WA and the Therapeutic Claims Committee of the International Federation of Multiple Sclerosis Societies. Therapeutic claims in multiple sclerosis. New York, NY Demos 1988

2. Kurtzke J. Rating neurologic impairment in multiple sclerosis: an expanded disability status scale (EDSS). Neurology 1983;33:1444-1452.

3. Cutter GR, Baier ML, Rudick RA, Cookfair DL, Fischer JS, Petkau J. et al. Development of a multiple sclerosis functional composite as a clinical trial outcome measure. Brain 1999;122:871-882.

4. Katz D, Taubenberger JK, Canella B, McFarlin DE, Raine CS, McFarland HF. Correlation between magnetic resonance imaging findings and lesion development in chronic, active multiple sclerosis. Ann Neurol 1993;34:661669.
5. Guttmann CR, Ahn SS, Hsu L, Kikinis R, Jolesz FA. The evolution of multiple sclerosis lesions on serial MR. AJNR Am J Neuroradiol 1995;16:1481-1491.

6. Rudick R, Fischer E, Lee J et al and the Multiple Sclerosis Collaborative Research Group. Use of the brain parenchymal fraction to measure whole brain atrophy in relapsing -remitting MS. Neurology 1999;52:1698-1704.

7. Johnson KP, Brooks BR, Cohen JA et al. Copolymer 1 reduces relapse rate and improves disability in relapsing -remitting multiple sclerosis: results of a phase III multicenter, double-blind, placebo-controlled trial. Neurology 1995;45:1268-1276.

8. PRISMS Study Group. Randomized double-blind, placebo-controlled study of interferon $\beta$-1a in relapsing/remitting multiple sclerosis. Lancet 1998;352:1498-1504.

9. Lublin FD, Reingold SC, and the National Multiple Sclerosis Society (USA) Task Force on Placebo-Controlled Clinical Trials in MS. Placebo-controlled clinical trials in multiple sclerosis: ethical consideration. Ann Neurol 2001;49:677-681.

10. Polman $\mathrm{CH}$, Reingold SC, Barkhof F, Calabresi PA, Clanet $\mathrm{M}$, Cohen JA et al. Ethics of placebo-controlled clinical trials in multiple sclerosis: a reassessment. Neurology 2009;70:1134-1140.

11. Su KG, Banker G, Bourdette D, Forte M. Axonal degeneration in multiple sclerosis: the mitochondrial hypothesis. Curr Neurol Neurosci Rep 2009;9:411-417.

12. Coyle PK. Early treatment of multiple sclerosis to prevent neurologic damage. Neurology 2008;71:S3-S7.

13. Trapp BD, Peterson J, Ranshoff RM, Rudick R, Mőrk S, Bő L. Axonal transection in the lesions of multiple sclerosis. N Engl J Med 1998;5:278-285.

14. Mainero C, Benner T, Radding A, van der Kouwe A, Jensen $R$, Rosen $B R$, Kinkel RP. In vivo imaging of cortical pathology in multiple sclerosis using ultra-high field MRI. Neurology 2009;12:941-948.

15. Rudick RA, Trapp BD. Gray-matter injury in multiple sclerosis. N Engl J Med 2009;361:1505-1506.

16. Fischer E, Lee JC, Nakamura K, Rudick RA. Gray matter atrophy in multiple sclerosis: a longitudinal study. Ann Neurol 2008;64:255-265.

17. Chard DT, Miller DH. What you see depends how you look: gray matter lesions in multiple sclerosis. Neurology 2009;73:918-919.

Acceptance for editing: 2015-04-29 Acceptance for publication: 2015-05-28

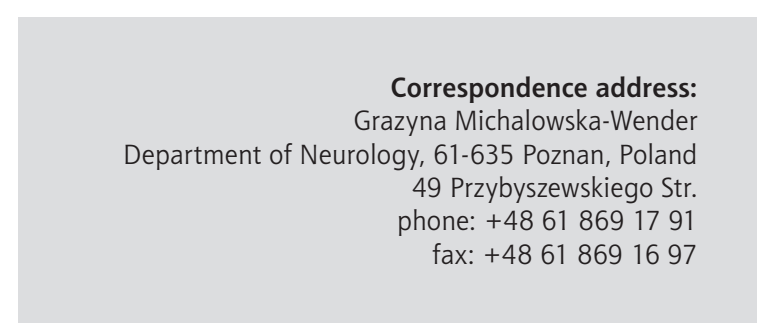

